\title{
Correlation between micro and macrostructural biaxial behavior of ascending thoracic aneurysm: a novel experimental technique
}

\author{
Emanuele Vignali ${ }^{\mathrm{a}, \mathrm{b}}$, Francesco di Bartolo ${ }^{\mathrm{a}, \mathrm{b}}$, Emanuele Gasparotti ${ }^{\mathrm{a}, \mathrm{b}}$, Antonio Malacarne ${ }^{\mathrm{c}}$, \\ Giovanni Concistréd, Francesca Chiaramonti ${ }^{\mathrm{d}}$, Michele Murzi ${ }^{\mathrm{d}}$, Vincenzo Positano ${ }^{\mathrm{a}}$, \\ Luigi Landini ${ }^{\mathrm{b}}$, Simona Celi ${ }^{\mathrm{a}, *}$ \\ a BioCardioLab, Ospedale del Cuore, Fondazione Toscana G. Monasterio, Massa, Italy \\ ${ }^{\mathrm{b}}$ Department of Information Engineering, University of Pisa, Pisa, Italy \\ ' Sant'Anna School of Advanced Studies, TeCIP Institute, Pisa, Italy \\ ${ }^{\mathrm{d}}$ Adult Cardiosurgery Unit, Ospedale del Cuore, Fondazione Toscana Gabriele Monasterio, Massa, Italy
}

\section{A R T I C L E I N F O}

\section{Article history:}

Received 15 March 2020

Revised 1 October 2020

Accepted 21 October 2020

\section{Keywords:}

Ascending thoracic aneurysm

Mechanical characterization

SALS

Microstructure

Collagen

\begin{abstract}
A B S T R A C T
Mechanical properties and microstructural modifications of vessel tissues are strongly linked, as established in the state of the art of cardiovascular diseases. Techniques to obtain both mechanical and structural information are reported, but the possibility to obtain real-time microstructural and macrostructural data correlated is still lacking. An experimental approach to characterize the aortic tissue is presented. A setup integrating biaxial traction and Small Angle Light Scattering (SALS) analysis is described. The system was adopted to test ex-vivo aorta specimens from healthy and aneusymatic (aTAA) cases. A significant variation of the fiber dispersion with respect to the unloaded state was encountered during the material traction. The corresponding microstructural and mechanical data were successfully used to fit a given anisotropic constitutive model, with satisfactory $R^{2}$ values $(0.97 \pm 0.11$ and $0.96 \pm 0.17$, for aTAA and healthy population, respectively) and fiber dispersion parameters variations between the aTAA and healthy populations $(0.39 \pm 0.23$ and $0.15 \pm 0.10)$. The method integrating the biaxial/SALS technique was validated, allowing for real-time synchronization between mechanical and microstructural analysis of anisotropic biological tissues.
\end{abstract}

(c) 2020 IPEM. Published by Elsevier Ltd. All rights reserved.

\section{Introduction}

The ascending thoracic aortic aneurysm (aTAA) pathology remains a clinical challenge requiring a deep insight concerning its formation and progression mechanisms. In fact, the nature of the aTAA disease remains silent, until the rupture occurs which is usually followed by the patient sudden death. From a biomechanical point of view, the critical state of an aTAA case arises from the status of the biological tissue and its degradation [1]. The mechanical properties of the aTAA depend on the constitutive microstructural components, which are given by collagen fiber bundles. The analysis of the tissue response is determined by the correlation between the microstructural components and the tensile state of the specimen. Several works concerning the characterization of the aTAA tissue, both in terms of structural and mechanical features, can be found in literature. Different methodologies for in-vivo

\footnotetext{
* Corresponding author.

E-mail addresses: s.celi@ftgm.it, simonaceli@libero.it (S. Celi).
}

[2] and ex-vivo [3] tissue testing were proposed. Uniaxial traction tests are widely used for aTAA tissue evaluation [4], however their contribution presents limitations due to the intrinsic anisotropic nature of the biological materials [5]. The gold standard technique in the context of mechanical evaluation is given by biaxial traction tests [6]. The complete characterization of the biaxial tensile traction allows the adoption of fiber based anisotropic constitutive equations, which represent the current state of the art models for aTAAs [7,8]. These constitutive formulations account for the presence of collagen fiber bundles, which are the main responsible for the macroscopic response of the vessel structures. On the basis of these evidences, a strong link between microstructure and macrostructure is established and the internal composition of the tissue cannot be neglected [9]. Concerning the evaluation of the microstructural fiber distribution, numerous techniques were adopted in the state of the art: small angle X-ray scattering [10], second harmonic generation microscopy [11], histologic analysis [12], diffusion tensor imaging [13] and multi-photon excited fluorescence [14]. The reported techniques can provide accurate quantitative information on fiber structure, however sometimes high 
a)

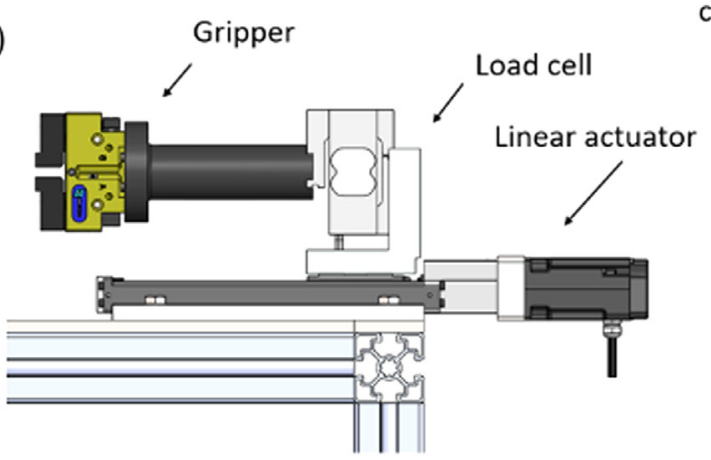

b)

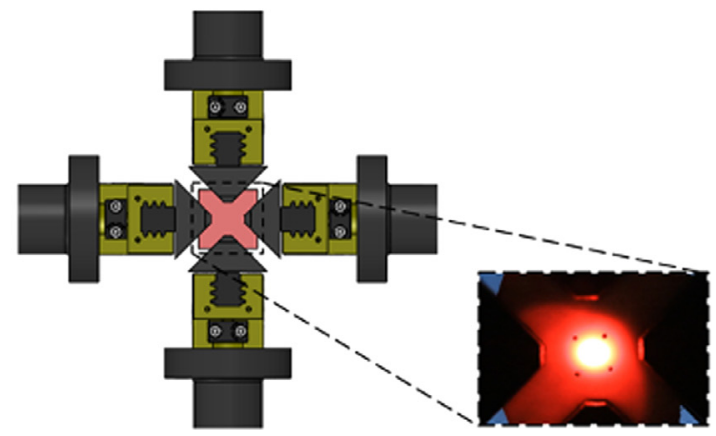

c)

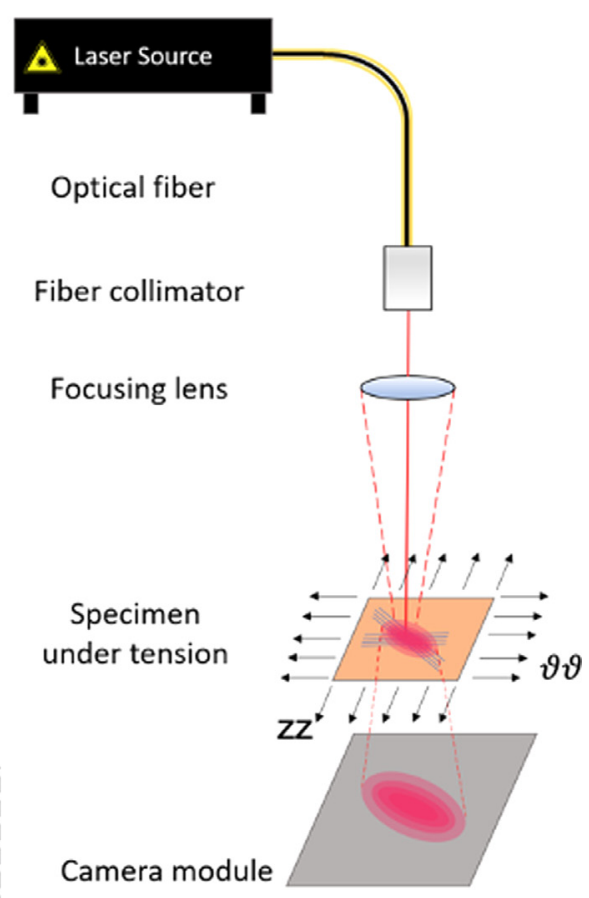

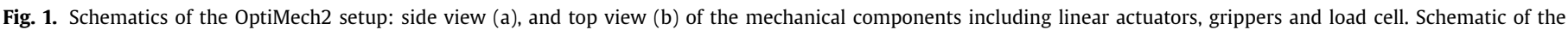
optical laser line (c).

energies are involved or they have an intrinsic destructive nature for the specimen. The adoption of energy sources like X-rays has the disadvantage of being a ionizing medium and, consequently, potentially harmful. In general, the optical approaches reported are not suitable for simultaneous macrostructural evaluation of the tissue specimens due to their complexity. The main objective would be to have a technique permitting the real-time synchronization of both the optical/microstructural and mechanical/macrostructural analysis, to have an evaluation of the microstructure modification as a consequence of the stress status of the material. The Small Angle Light Scattering (SALS) is an optical, non-destructive technique able to provide semi-quantitative structural information about fibrous structures by using a low energy laser source and evaluating the corresponding scattered pattern after the specimen irradiation [15]. The SALS method was already used for vessel tissue evaluation in previous works [16]. The information regarding the tissue microstructure was correlated to the tension state of the specimen, but the analysis was limited to uniaxial load and it was not possible to evaluate the tissue anisotropy.

Currently, there are no studies providing real-time synchronization with tensile biaxial data and microstructural information for vascular tissue analysis. On the basis of the current background, the aim of this work is to present the characterization of biological aTAA tissues through an innovative experimental setup integrating the biaxial traction procedure of the specimen with an optical SALS analysis to assess the microstructure. First, an overview of the experimental setup is presented. Then, the system was adopted to test ex-vivo aorta specimens from healthy and aTAA porcine cases. Both the stress/stretch and microstructural results are reported. In particular, the experimental curves obtained from both the biaxial traction and SALS analysis were adopted for the data fitting through a state of the art constitutive model. The proposed model accounts for the presence of microstructural components by including within the strain energy density function the contribution of two main families of collagen fibers with dispersion. Particular attention was given to the evaluation of the fiber distribution modification as a function of the tensional state.

\section{Materials and methods}

\subsection{Experimental setup definition}

A specific custom setup was developed, in which simultaneous biaxial traction and SALS analysis were carried out (OptiMech2). In particular, the biaxial traction procedure is imposed with a controllable testing machine, already described in previous publications [8,17]. The schematic of the different components is reported in Fig. $1 \mathrm{a}$ and b. In synthesis, the machine is constituted by two linear axes actuated by servomotors. The configuration allows soft specimen traction along two directions, which for the specific case of aortic specimens are given by the circumferential $(\theta \theta)$ and longitudinal directions $(z z)$ (see also Fig. 3). Pneumatic holders with modular 3D printed grippers were designed to assure the specimen binding. The corresponding forces are recorded through two dedicated strain-gauge load cells, with a force accuracy of $0.1 \mathrm{~N}$ and full scale range of $50 \mathrm{~N}$. The deformations are acquired through an optical system given by a CCD camera module (resolution $0.03 \mathrm{~mm} / \mathrm{pixel}$ ) adopted for the tracking of four calibrated markers applied on the specimen surface. A tensioning protocol can be imposed to control the axes movement and to maintain the load status of the specimen. Different constant tension ratios $\left(T_{\theta \theta}: T_{z z}\right)$ are applied during the traction.

A schematic of the optical part is illustrated in Fig. 1 c. The system is constituted by an unpolarized He-Ne laser source (wavelength $\lambda=632.8 \mathrm{~nm}$, power $\mathrm{P}=5 \mathrm{~mW}$ ) directly connected to an optical fiber with a collimator. The laser beam is focused on the specimen through a bi-focal lens. The SALS laser pattern is acquired through the same camera system already adopted for the marker tracking. The test procedure and data acquisition are obtained through a dedicated real-time processor (NI cDAQ-9132), 

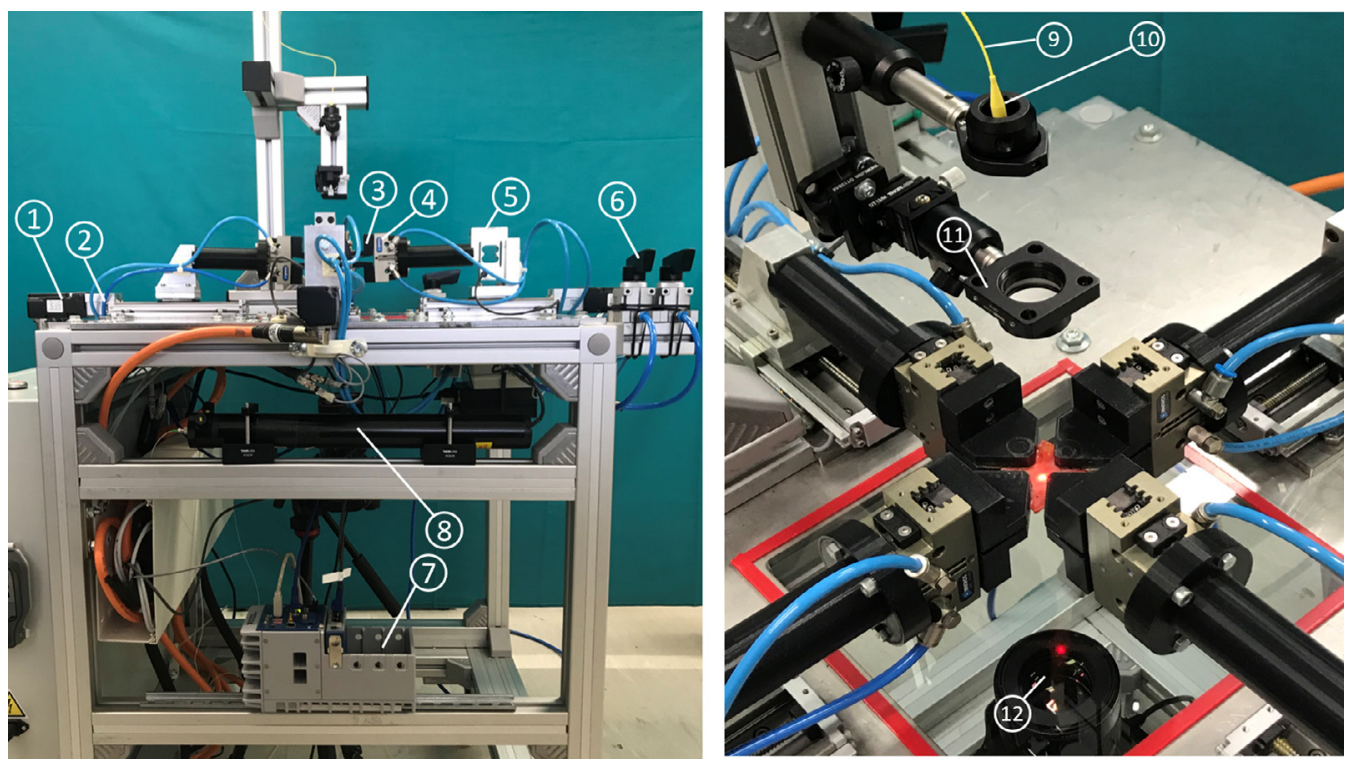

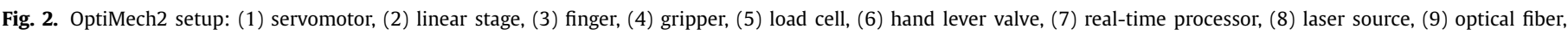
(10) collimator, (11) focusing lens and (12) camera.

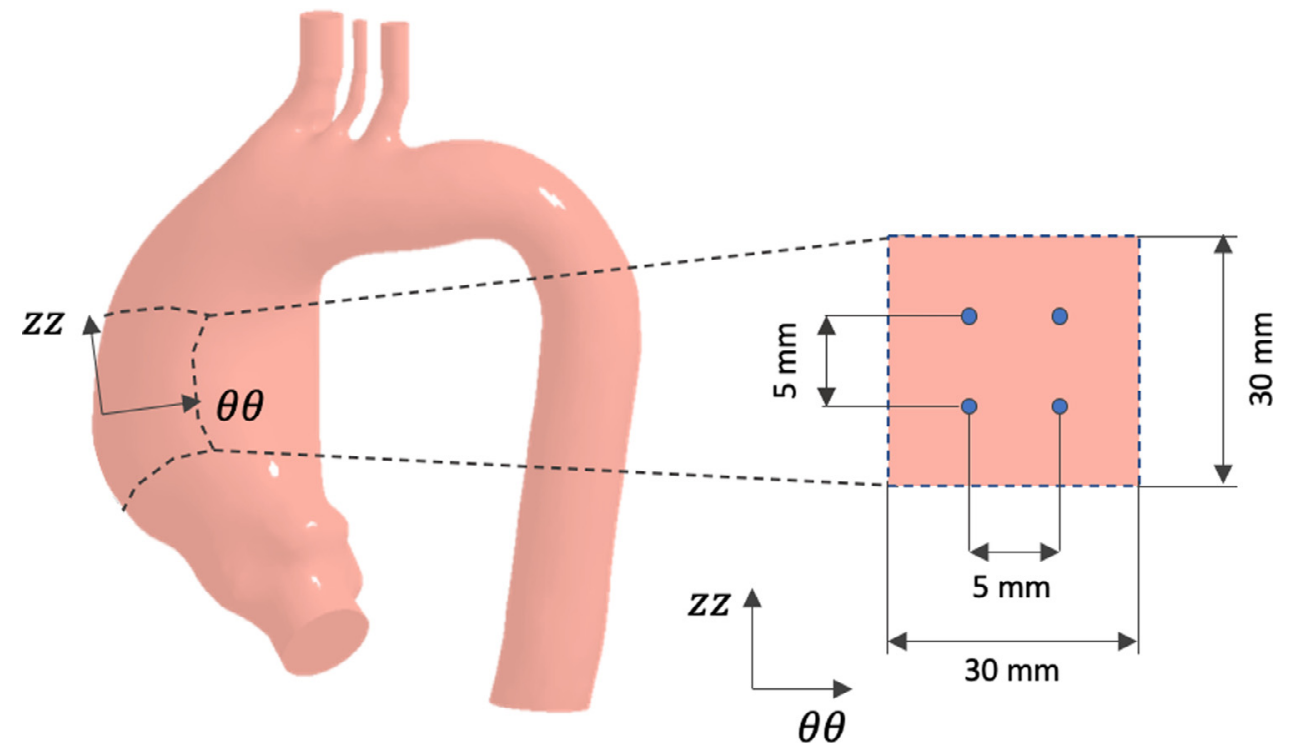

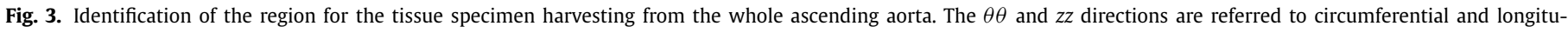
dinal directions, respectively.

controlled through a custom LabView application. The complete OptiMech2 setup realized is reported in Fig. 2.

\subsection{Tissue preparation}

A population of tissue specimens was gathered to be tested with the OptiMech2 experimental setup. In particular, the tissues were retrieved from a local slaughterhouse from which whole ascending aorta sections were harvested from swines. In order to assess the health status of the aortic tissue, the study relied on veterinary support. Additionally, the diameter at the ascending section level was measured. All the cases in which the diameter value was $50 \%$ above the baseline range of a healthy adult swine were assumed as aTAA. Three healthy and five aTAA specimens were obtained. A square specimen of about $30 \mathrm{~mm}$ side was cut in the outer curvature region (Fig. 3) to limit the regional variation influence [18-20]. To assure the tissue freshness, the specimen was maintained at room temperature in physiological solution (saline solution $\mathrm{NaCl} 0.9 \%$ ) and tested at maximum 3 hours after tissue harvesting from ex-vivo aorta. No freezing process was imposed to preserve the microstructure integrity [21].

\subsection{Test procedure}

For each specimen, the mechanical test consisted in a biaxial traction sequence with five different $T_{\theta \theta}: T_{z z}$ ratios (1:1, 0.5:1, $0.75: 1,1: 0.5,1: 0.75)$. The traction process was configured according to a tension control algorithm, designed to reach the maximum physiological load, set to $120 \mathrm{~N} / \mathrm{m}$ according to previous literature studies [22]. The load was applied by imposing a quasi-static tensioning protocol with tension set-points increase of $3.3 \mathrm{~N} / \mathrm{m}$ and a maximum rate of $6.0 \mathrm{~N} / \mathrm{m} / \mathrm{s}$. Prior to collecting data, the tensioning protocol was used to impose nine loading and unloading cycles of biaxial preconditioning for each specimen with a pre-load of $0.2 \mathrm{~g}$. 
Mechanical data were reported in terms of stress-stretch curves along the $\theta \theta$ and $z z$ directions $\left(\sigma_{\theta \theta}-\lambda_{\theta \theta}\right.$ and $\left.\sigma_{z z}-\lambda_{z z}\right)$. Specific calibrated markers (diameter equal to $1.4 \mathrm{~mm}$ ) were placed in the center of the specimen in order to form a square with $5 \mathrm{~mm}$ side (Fig. 3). The marker positions were used to evaluate the stretches as relative displacements measurements. By assuming a planar biaxial tensioning of an incompressible square flat specimen [23], the Cauchy stress values were evaluated as:

$\sigma_{i i}=\left(F_{i i} / A_{0}\right) \lambda_{i i}$

where $i$ represents the generic testing direction, $F_{i i}$ represents the tensioning force recorded by the load cells and $A_{0}$ represents the unloaded specimen cross-section. Microstructural data were acquired on the basis of the resulting analysis of the SALS pattern. In particular, given the characteristic specimen thickness of $1.48 \pm 0.47 \mathrm{~mm}$, the pattern was interpreted as an ellipses deriving from multiple scattering phenomena [24]. The ellipses eccentricity variation $(\Delta E)$ and inclination angle $(\phi)$ were calculated during the traction procedure, given their link with fiber dispersion and preferred direction, respectively. The $\phi$ angle was referred to the circumferential direction [25]. Additionally, the fiber distribution during the test was evaluated by sampling the light intensity pattern along a circular profile with a radius given by the projection of the beam opening [15]. The data were normalized in order to have an unitary integral to permit the fitting with the symmetrical fiber families distribution.

\subsection{Constitutive modeling}

Macrostructural and microstructural data were used to impose a constrained fitting of a state of the art fiber-based constitutive model [26]. The model is given by the contribution of two fiber families with dispersion and it is defined according to the following constitutive equation:

$W=\frac{c}{2}\left(\mathbf{I}_{1}-3\right)+\frac{k_{1}}{2 k_{2}} \sum_{i=4,6}\left[e^{\left(k_{2}^{i}\left(\mathbf{I}_{i}^{*}-1\right)^{2}\right)}-1\right]$

where $c, k_{1}$ and $k_{2}$ are the constitutive model mechanical parameters representing the matrix stiffness, the fiber stiffness and the fiber stiffening factor, respectively. The $\mathbf{I}_{1}$ is the first right CauchyGreen strain tensor invariant and $\mathbf{I}_{i}^{*}$ is the pseudo invariant defined on the basis of the generalized structure tensor $\mathbf{H}_{i}$, given by:

$\mathbf{H}_{i}=A \mathbf{I}+B \mathbf{M}_{i} \otimes \mathbf{M}_{i}+(1-3 A-B) \mathbf{M}_{o p} \otimes \mathbf{M}_{o p} \quad i=4,6$

where $\mathbf{M}_{o p}$ is the out of plane direction vector and $\mathbf{M}_{i}$ is the vector defining the $i$-th family preferential direction. The fiber preferential direction is assumed to lie in the $\theta \theta-z z$ plane, defined, respectively, by the versors $\mathbf{e}_{1}$ and $\mathbf{e}_{2}$. Consequently, $\mathbf{M}_{i}$ is completely defined by the fiber family angle parameter $\alpha_{i}$, according to:

$\mathbf{M}_{i}=\cos \alpha_{i} \mathbf{e}_{1}+\sin \alpha_{i} \mathbf{e}_{2} \quad i=4,6$

It is worth to note that, if $\alpha_{i}=0^{\circ}$, the $\mathbf{M}_{i}$ vector is parallel to the $\theta \theta$ direction. The fiber dispersion is modeled in Eq. (3) through the parameters $A$ and $B$, which depend directly on the microstructural parameters $k_{i p}$ and $k_{o p}$, representing the in-plane and out-of-plane dispersion factors, according to:

$A=2 k_{i p} k_{o p}, \quad B=2 k_{o p}\left(1-2 k_{i p}\right)$

By starting from the expression of the strain energy density function reported in Eq. (2), it is possible to evaluate the stress tensor matrix of the constitutive model. The value calculated for the fitting with biaxial data is given by the Cauchy stress. The Cauchy stress matrix is expressed by:

$\boldsymbol{\sigma}=\frac{1}{J} \mathbf{F} \cdot\left(2 \frac{\delta W}{\delta \mathbf{C}}-p \mathbf{C}^{-1}\right) \cdot \mathbf{F}^{T}$ where $\boldsymbol{\sigma}$ is the Cauchy stress tensor, $J$ is the Jacobian, $\mathbf{F}$ is the deformation gradient, $\mathbf{C}$ is the right Cauchy-Green deformation tensor and $p$ is a Lagrangian constant to take into account for incompressibility. Under the assumption of purely biaxial stress on an incompressible tissue, the normal circumferential $\left(\sigma_{\theta \theta}\right)$ and longitudinal $\left(\sigma_{z z}\right)$ stress are non-null while the radial stress $\left(\sigma_{r r}\right)$ is imposed as null to evaluate the value of the scalar $p$. For the specific case of the $W$ expressed in Eq. (2) and by considering the derivative form in Eq. (6), the expression of Cauchy stresses is given by:

$\sigma_{\theta \theta}=c\left(\lambda_{\theta \theta}^{2}-\frac{1}{\lambda_{\theta \theta} \lambda_{z z}}\right)+4 k_{1}\left(I_{4}^{*}-1\right) e^{\left(k_{2}\left(I_{4}^{*}-1\right)^{2}\right)} \mathbf{H}_{4}(1,1)-p$

$\sigma_{z z}=c\left(\lambda_{z z}^{2}-\frac{1}{\lambda_{\theta \theta} \lambda_{z z}}\right)+4 k_{1}\left(I_{4}^{*}-1\right) e^{\left(k_{2}\left(I_{4}^{*}-1\right)^{2}\right)} \mathbf{H}_{4}(2,2)-p$

where, according to fiber symmetry distribution assumption, the $I_{4}^{*}=I_{6}^{*}$ and $\mathbf{H}_{4}=\mathbf{H}_{6}$ conditions hold. The $p$ scalar value of the Lagrangian is set according to the following equation, obtained by imposing the $\sigma_{r r}=0$ condition:

$p=4 k_{1}\left(I_{4}^{*}-1\right) e^{\left(k_{2}\left(I_{4}^{*}-1\right)^{2}\right)} \mathbf{H}_{4}(3,3)$

The expressions from Eq. (7) are used to fit the experimental data from the testing procedures. The fitting procedure was set in order to minimize the following score expression:

$J_{\text {score }}=\sum_{i=1}^{N_{s}}\left[\sigma_{i}-\tilde{\boldsymbol{\sigma}}_{i}\right]^{2}$

where $\sigma_{i}$ is the stress tensor, $\tilde{\boldsymbol{\sigma}}_{i}$ is the stress tensor from the experimental stress data and $N_{S}$ is the number of experimental samples. In synthesis, the model is completely defined by a set of seven parameters, which are both mechanical $\left(c, k_{1}, k_{2}\right)$ and microstructural $\left(\alpha_{4,6}, k_{i p}, k_{o p}\right)$. The fiber distribution from the SALS analysis was used to constrain the search space of the microstructural parameters $\alpha_{4,6}$ and $k_{i p}$. In particular, the SALS distribution was interpreted through the superimposition of two Von Mises Distributions (VMDs), representing the fiber families. The VMDs are assumed to have the same dispersion and to be symmetrical around the $\phi$ angle. The constitutive model parameters were expressed according to:

$\alpha_{i=4,6}=\phi \pm \eta_{i=4,6}, \quad k_{i p}=\frac{1}{2}-\frac{I_{1}(a)}{2 I_{0}(a)}$

Where $\eta_{i=4,6}$ and $a$ are the mean values and variance parameter of the VMDs, respectively. The functions $I_{0}(x)$ and $I_{1}(x)$ are given by the modified Bessel functions of zero and first order. The estimations from the VDMs from SALS patterns were used to constrain the search space for the corresponding parameters around $\pm 10 \%$ of the values from expression in Eq. (6). The fitting process was carried out on the basis of these constraints and by using a TrustRegion-Reflective algorithm [27], implemented through a custom MATLAB script to minimize the expression reported in Eq. (9). The overall fit scores were evaluated through the determination coefficient $\left(\hat{R^{2}}\right)$ and the Normalized Root-Mean-Square Error (Err). Additionally, the $R^{2}$ values specific for each tension ratio imposed were calculated.

\section{Results}

The testing procedure, including both the mechanical and optical part, was carried out with success through the OptiMech2 setup. The constrained fitting process on the basis of the presented constitutive model was obtained and the relative coefficients are reported in Table 1 for both the healthy and the aTAA whole populations. To evaluate the trend of the fitting process on the validation set, the values are reported as mean \pm standard deviation. 
Table 1

Mechanical and microstructural coefficients from the constrained fitting of constitutive model imposed on the population of aTAA and healthy cases (mean \pm standard deviation).

\begin{tabular}{llllllll}
\hline & $c(k P a)$ & $k_{1}(k P a)$ & $k_{2}$ & $\alpha_{4}\left({ }^{\circ}\right)$ & $\alpha_{6}\left({ }^{\circ}\right)$ & $k_{i p}$ & $k_{o p}$ \\
\hline aTAA & $18.6 \pm 56.2$ & $384 \pm 378$ & $14.3 \pm 32.1$ & $5.4 \pm 2.7$ & $-16.3 \pm 9.0$ & $0.39 \pm 0.23$ & $0.31 \pm 0.17$ \\
Healthy & $23.6 \pm 35.7$ & $21.1 \pm 17.7$ & $1.9 \pm 8.9$ & $0.1 \pm 3.4$ & $-6.9 \pm 7.7$ & $0.15 \pm 0.10$ & $0.47 \pm 0.27$ \\
\hline
\end{tabular}
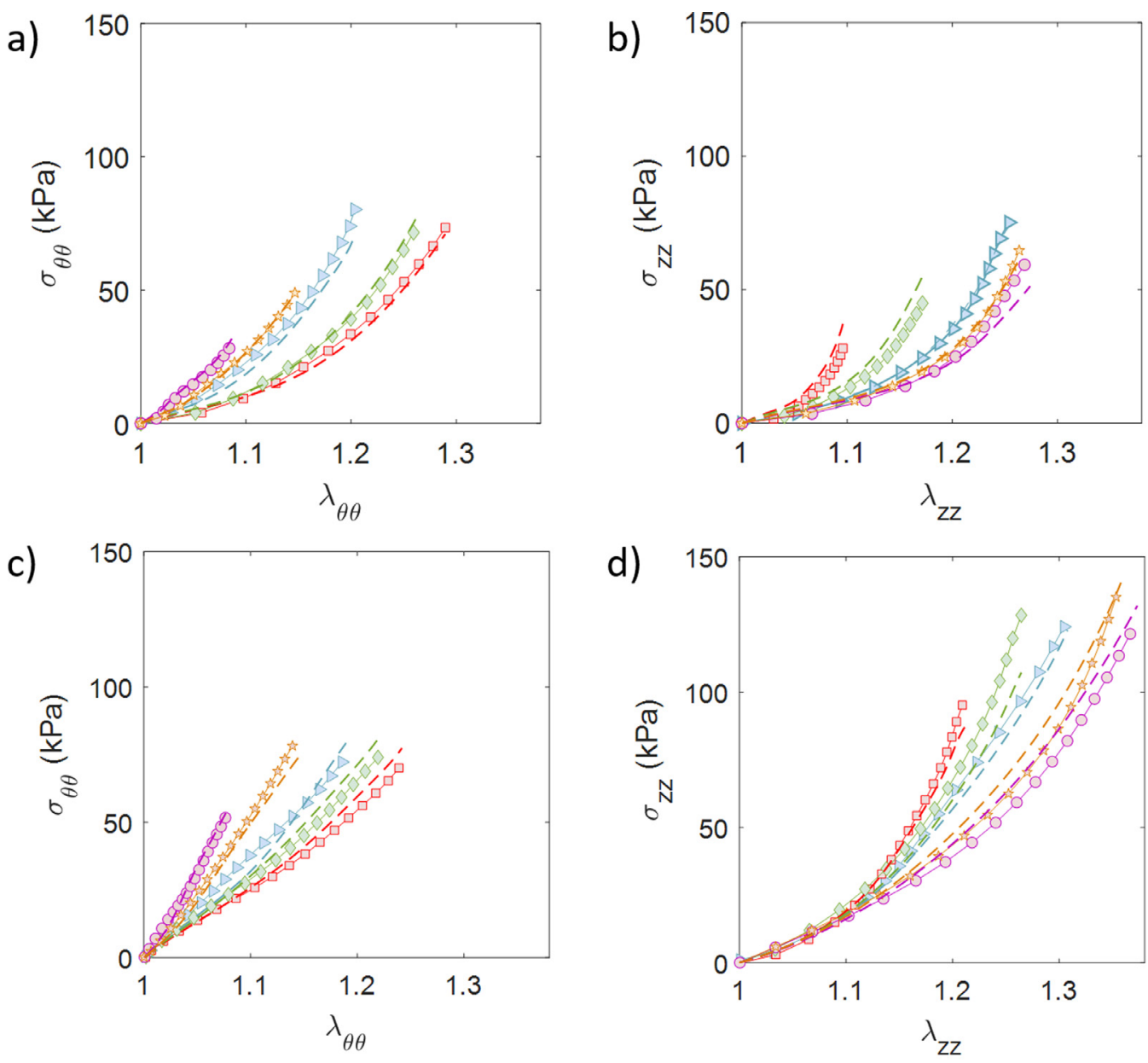

\begin{tabular}{cccccc|}
$T_{\theta \theta}: T_{z Z}$ & $1: 1$ & $1: 0.5$ & $1: 0.75$ & $0.5: 1$ & $0.75: 1$ \\
Exp. & - & $-\square-$ & - & -0 & 年 \\
Fit. & --- & --- & --- & --- & ---
\end{tabular}

Fig. 4. Tensile data in terms of circumferential $\left(\sigma_{\theta \theta}-\lambda_{\theta \theta}\right)$ and longitudinal $\left(\sigma_{z z}-\lambda_{z z}\right)$ stress-stretch for the aTAA (a-b) and the healthy example case (c-d).

A set of exemplificative cases, including an healthy and an aTAA, is reported in Fig. 4 and Fig. 5, both in terms of mechanical and microstructural data, respectively. The $R^{2}$ values for each tension ratio considered and the overall $\hat{R}^{2}$ and Err scores are reported in Table 2. The mean overall $\hat{R^{2}}$ value was equal to $0.96 \pm 0.11$ for healthy and $0.97 \pm 0.17$ for aTAA. The scores reported revealed the effectiveness of the fitting process, with satisfactory values of determination coefficients and negligible errors for each tension ratio considered. The lowest scores were encountered for the ratio biased towards the $z z$ direction for the healthy population. For the $0.5: 1$ ratio, the scores were $0.96 \pm 0.12$ and $0.97 \pm 0.14$ for $\sigma_{\theta \theta}$ and $\sigma_{z z}$, respectively; while for $0.75: 1$, scores of $0.96 \pm 0.15$ and $0.97 \pm 0.10$ were calculated. Nevertheless the values were always above the 0.95 value and the trend was correctly coped. The microstructural data from the SALS pattern, reported in Fig. 5, appeared to be strongly influenced by the loading status of the tissue.
Table 2

Scores for the constrained fitting in terms of $R^{2}$ evaluated on different tension ratios stress-stretch curves and of overall $\hat{R}^{2}$ and Err (mean \pm standard deviation).

\begin{tabular}{|c|c|c|c|c|}
\hline \multirow{2}{*}{$T_{\theta \theta}: T_{z z}$} & \multicolumn{2}{|l|}{ aTAA } & \multicolumn{2}{|l|}{ healthy } \\
\hline & $\theta \theta$ & $z z$ & $\theta \theta$ & $z z$ \\
\hline $1: 1$ & $0.99 \pm 0.12$ & $0.99 \pm 0.12$ & $0.97 \pm 0.10$ & $0.98 \pm 0.10$ \\
\hline $1: 0.5$ & $0.97 \pm 0.11$ & $0.98 \pm 0.12$ & $0.97 \pm 0.13$ & $0.99 \pm 0.13$ \\
\hline $1: 0.75$ & $0.98 \pm 0.15$ & $0.98 \pm 0.11$ & $0.98 \pm 0.10$ & $0.98 \pm 0.12$ \\
\hline $0.5: 1$ & $0.99 \pm 0.13$ & $0.98 \pm 0.11$ & $0.96 \pm 0.12$ & $0.97 \pm 0.14$ \\
\hline $0.75: 1$ & $0.99 \pm 0.11$ & $0.99 \pm 0.13$ & $0.96 \pm 0.15$ & $0.97 \pm 0.10$ \\
\hline$\hat{R^{2}}$ & \multicolumn{2}{|c|}{$0.97 \pm 0.11$} & \multicolumn{2}{|c|}{$0.96 \pm 0.17$} \\
\hline Err & \multicolumn{2}{|c|}{$1.1 \pm 2.4 \%$} & \multicolumn{2}{|c|}{$1.0 \pm 3.1 \%$} \\
\hline
\end{tabular}

In particular, the ratios biased along $\theta \theta$ direction $(1: 0.5,1: 0.75)$ revealed a significant $\Delta E$ increase for both cases represented. Con- 


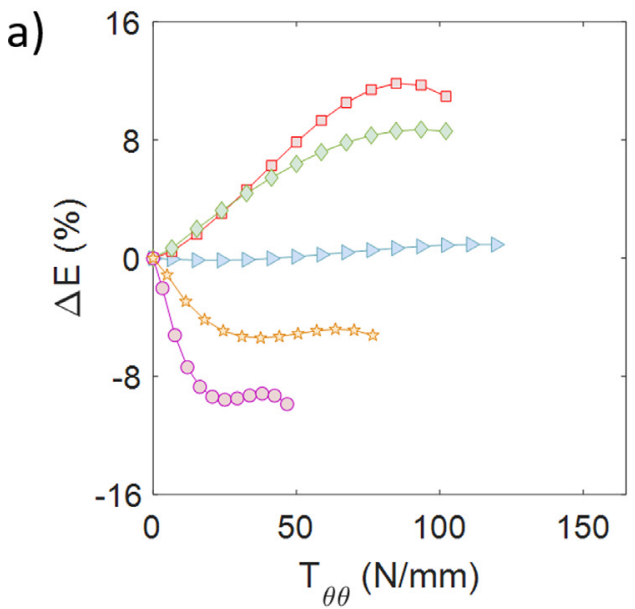

c)

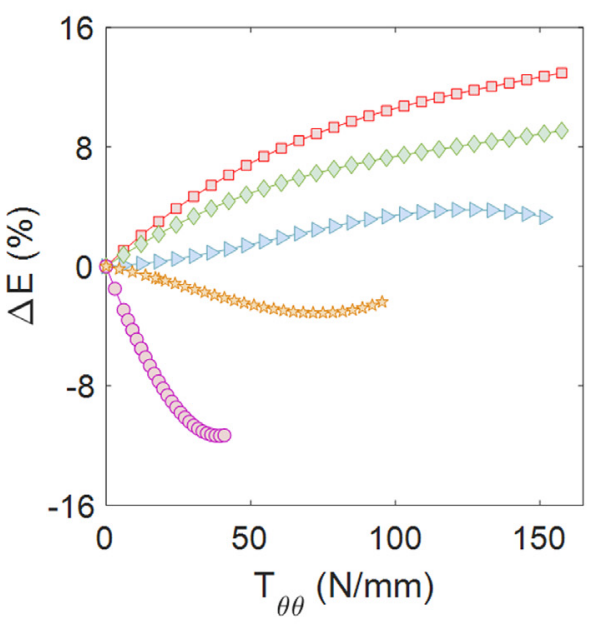

b)

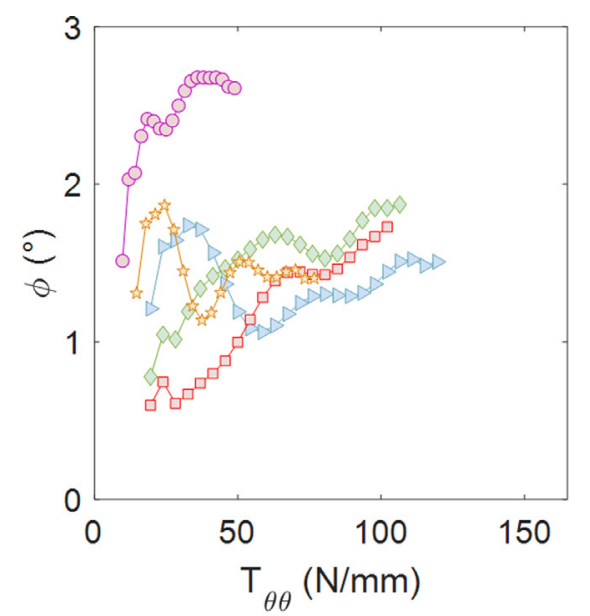

d)

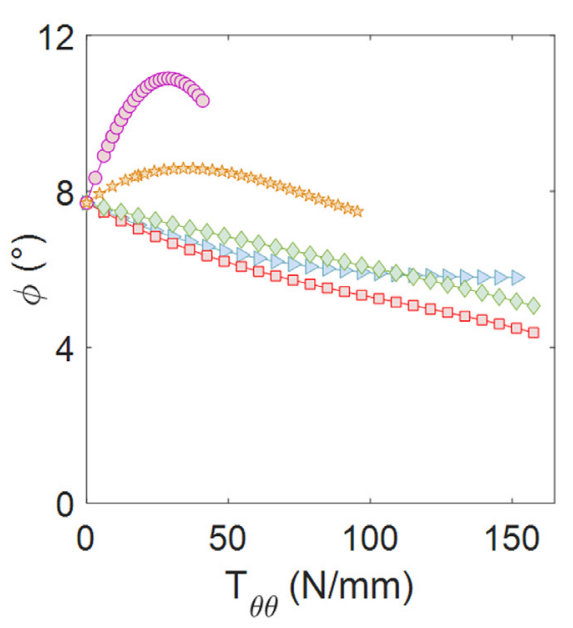

\begin{tabular}{cccccc|}
$T_{\theta \theta}: T_{z Z}$ & $1: 1$ & $1: 0.5$ & $1: 0.75$ & $0.5: 1$ & $0.75: 1$ \\
Exp. & - & $\square-$ & - & $-0-$ & - b
\end{tabular}

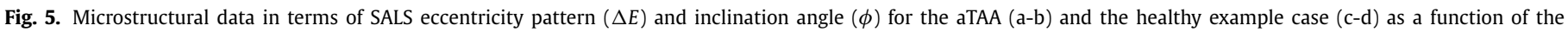
imposed tension.

cerning the $\phi$ values, a clear decrease is present in the healthy case, while the behavior in the aTAA is approximately constant. Both the angle and the eccentricity variations can be interpreted by considering the fiber families progressive alignment along the $\theta \theta$ direction as a consequence of tensioning. The ratios biased towards the $z z$ direction $(0.5: 1,0.75: 1)$ exhibited a more complex trend. While for both the aTAA and the healthy case, the eccentricity diminishes, the $\phi$ value showed a rising behavior with a peak and a consequent decrease for the healthy case only. It is possible to assume that the microstructure encountered a simultaneous fiber families rotation and reorganization caused by the biased tension states of $0.5: 1$ and $0.75: 1$.

The fiber distributions, evaluated at different tension ratios, together with the fiber families VMDs, are reported in Fig. 6. In particular, the results from the equibiaxial and the extreme ratios ( $1: 0.5$ and $0.5: 1$ ) were compared with the unloaded state. It is possible to note that the fitting through the superimposition of two VMDs was successful. A significant difference in terms of distribution variance emerged from the comparison between the aTAA and the healthy cases. The pathological case revealed a more dispersed behavior, while the fibers appeared to be more aligned in the healthy case. Additionally, it was possible to denote the effects of the different tensioning protocols. It appears that the equibiaxial tension slightly effected the fiber distribution in both cases, with a angle shift towards the $\theta \theta$ direction corresponding to $0^{\circ}$. On the contrary, the 1:0.5 and 0.5:1 ratios strongly influenced the fiber organization. In particular, the dispersion encountered a significant rise when the tension is biased towards the $\theta \theta$ direction. In this case, a significant alignment was assumed to occur. In opposition to this trend, the dispersion decreased for the $0.5: 1$ ratio. For this last case, the families appeared as almost collapsed, as it is possible to determine from the corresponding VMDs.

\section{Discussion}

The results presented in the previous section demonstrated the effectiveness of the method combining the optical and tensile investigation of SALS and biaxial traction techniques. As the microstructural and macrostructural aspects of the aTAA tissue evaluation are highly correlated, the development of this new setup, permitting the synchronized analysis of the two aspects, would be a valuable addition to the context of aneurysmatic soft tissues. The adopted material model, accounting for the identified arrangement of collagen fibers, was capable of providing good fits for the samples although parameters constraints were present. The adoption of the constraint based on the microstructural parameters did not negatively influence the fitting performance, as both the overall and the tension-ratio specific $R^{2}$ scores revealed remarkable val- 

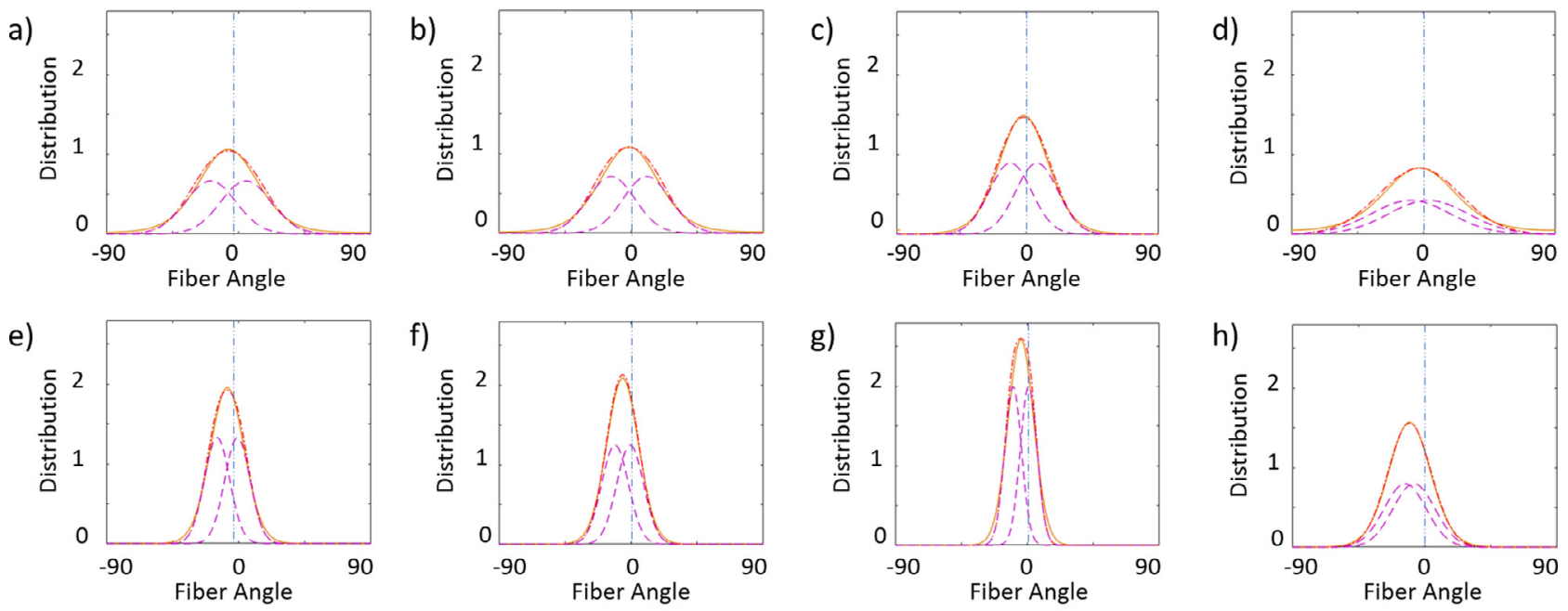

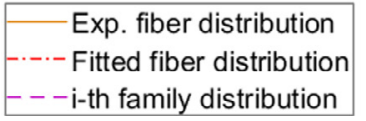

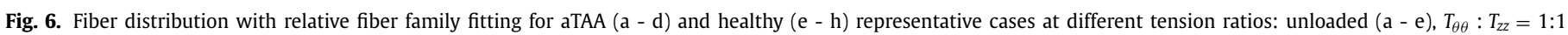
(b-f), $T_{\theta \theta}: T_{z z}=1: 0.5(\mathrm{c}-\mathrm{g})$ and $T_{\theta \theta}: T_{z z}=0.5: 1(\mathrm{~d}-\mathrm{h})$.

ues $\left(R^{2}>0.95\right)$, even for the more biased ratios. Moreover, fiber dispersion variations between the aTAA and healthy populations $(0.39 \pm 0.23$ and $0.15 \pm 0.10)$ were encountered. The distribution of the microstructural components appeared to be influenced deeply by the different tension states of the specimen, as confirmed by the dynamical data from the SALS analysis.

The method of SALS applied for the evaluation of strains within the context of soft biological tissues was established already [25]. The group of Gaul et al. reported the usage of SALS characterization of strain-induced degradation of collagenous structures within the ex-vivo arterial tissues $[16,28]$. Nevertheless, the interest of the study was limited to uniaxial traction. Additionally, the test procedure was mainly carried out by imposing strain set points at which the sample was held during the optical characterization by SALS. No control of the tension state of the specimen under analysis was reported. The further step provided by the development of the OptiMech2 setup is to determine a fully biaxial deformation patterns in real-time and by imposing a controlled tension protocol. The proposed setup was demonstrated to be unique and able to register both macrostructural and microstructural data in terms of stress/stretch curves and SALS pattern modifications. To our knowledge, this is the first time biaxial and microstructural data are presented together in correlation at different tension ratios. Additionally, the gathered experimental results were used for constrained constitutive modeling according to fiber-based strain density energy functions from the state of the art. The strength point of the proposed setup resides in the possibility to detect anisotropic effects thanks to the biaxial nature of the approach, providing real-time synchronization with the mechanical traction in a non-destructive manner.

The presented study has some points of possible improvement to be discussed. The main limitation of the SALS technique is that it is not suitable for the investigation of the fiber architecture along the specimen thickness. More accurate details on this aspect were provided by using a polarized light microscope [29], the second harmonic generation microscopy [30] or diffusion tensor imaging technique [31,32]. Despite this limitation, our results are in agreement with previous related works in terms of fiber distribution [11]. In fact, it is worth to stress out that the aneurysmatic tissues exhibited an increased fiber dispersion in comparison with the healthy cases. The population used for the study was limited by the tissue availability and it is not actually sufficient to be compared with other analyses in which wider samples were involved. Moreover, the study was limited to porcine cases, and it would be interesting to extend it to human specimens, as it was demonstrated that differences among the two models exist within this context [33]. Nevertheless, the validation of the methodology was successful and additional cases to be evaluated will be included during the further development of the experimental approach.

Even though numerous engineering/basic science works concerning the aTAA rupture issue are present in the state of the art, the risk evaluation strategy remains weak and further effort is still required. The characterization of aneurysm tissues remains a hot topic and a difficult task to fulfill, consequently alternative experimental setups [34] and new numerical approaches to overcome these challenges are currently investigated [35,36]. The combination of both optical and mechanical experimental facilities permitted to open new paths within this context. The proposed approach represents an an additional brick in the aTAA characterization landscape, for a more accurate and complete evaluation of the aneurysm disease from a microstructural and macrostructural point of view.

\section{Conclusion}

The importance of mechanical constitutive modeling in the analysis of aTAA was underlined in different studies in the state of the art. The presence and distribution of microstructural components, like collagen fibers, play a key role in the mechanical response of the aortic tissue. With this study, an approach for the simultaneous microstructural and macrostructural analysis of aortic tissue specimens was presented. The developed setup permitted the synchronous biaxial tension and SALS microstructure analysis of populations of pathological (aTAA) and healthy cases. The results were reported in terms of circumferential and longitudinal stress-stretch curves and microstructural parameters evaluated on the basis of SALS pattern analysis. The reported data demonstrated the successful development of the method. The fiber distribution evaluation from SALS analysis of specimens under biaxial tensioning was obtained. In particular, the imposed biaxial loads resulted 
to have a strong influence on the fiber redistribution inside the evaluated specimens. Additionally, the constitutive model fitting, obtained by setting microstructural parameters constraint from the SALS analysis, revealed remarkable scores. In conclusion, the combined SALS biaxial approach was developed successfully with the objective to fill the gap between structurally motivated constitutive models for aTAA and fiber distribution characterization. The study presented aims to be an innovative step in the landscape of aTAA macrostructural and microstructural analysis.

\section{Declaration of Competing Interest}

The authors declare no conflict of interest.

\section{Acknowledgments}

This work was funded by Fondazione Pisa through the project DIVINE (IFOPISA17AM).

\section{Ethical approval}

Not required

\section{References}

[1] Iliopoulos DC, Kritharis EP, Giagini AT, Papadodima SA, Sokolis DP. Ascending thoracic aortic aneurysms are associated with compositional remodeling and vessel stiffening but not weakening in age-matched subjects. J Thorac Cardiovasc Surg 2009;137(1):101-9.

[2] Fanni BM, Capellini K, Di Leonardo M, Clemente A, Cerone E, Berti S, et al. Correlation between LAA morphological features and computational fluid dynamics analysis for non-valvular atrial fibrillation patients. Appl Sci 2020;10(4):1448.

[3] Ferrara A, Morganti S, Totaro P, Mazzola A, Auricchio F. Human dilated ascending aorta: mechanical characterization via uniaxial tensile tests. J Mech Behav Biomed Mater 2016;53:257-71.

[4] Kritharis EP, Iliopoulos DC, Papadodima SA, Sokolis DP. Effects of aneurysm on the mechanical properties and histologic structure of aortic sinuses. Ann Thorac Surg 2014;98(1):72-9.

[5] Duprey A, Trabelsi O, Vola M, Favre J, Avril S. Biaxial rupture properties of ascending thoracic aortic aneurysms. Acta Biomater 2016;42:273-85.

[6] Trabelsi O, Davis FM, Rodriguez-Matas JF, Duprey A, Avril S. Patient specific stress and rupture analysis of ascending thoracic aneurysms. J Biomech 2015;48(10):1836-43.

[7] Holzapfel G, Niestrawska J, W Ogden R, Reinisch A, Schriefl A. Modelling non-symmetric collagen fibre dispersion in arterial walls. J R Soc Interface 2015;12.

[8] Vignali E, Gasparotti E, Capellini K, Fanni BM, Landini L, Positano V, et al. Modeling biomechanical interaction between soft tissue and soft robotic instruments: importance of constitutive anisotropic hyperelastic formulations. Int J Rob Res 2020. 0278364920927476

[9] Weisbecker H, Viertler C, Pierce DM, Holzapfel GA. The role of elastin and collagen in the softening behavior of the human thoracic aortic media. J Biomech 2013;46(11):1859-65.

[10] Schmid F, Sommer G, Rappolt M, Regitnig P, Holzapfel GA, Laggner P, et al. Bidirectional tensile testing cell for in situ small angle x-ray scattering investigations of soft tissue. Nucl Instrum Methods Phys Res, Sect B 2006;246(1):262-8.

[11] Niestrawska JA, Viertler C, Regitnig P, Cohnert TU, Sommer G, Holzapfel GA. Microstructure and mechanics of healthy and aneurysmatic abdominal aortas: experimental analysis and modelling. J R Soc Interface 2016;13(124):20160620.

[12] Pasta S, Phillippi JA, Tsamis A, D'Amore A, Raffa GM, Pilato M, et al. Constitutive modeling of ascending thoracic aortic aneurysms using microstructural parameters. Med Eng Phys 2016;38(2):121-30.

[13] Flamini V, Creane AP, Kerskens CM, Lally C. Imaging and finite element analysis: a methodology for non-invasive characterization of aortic tissue. Med Eng Phys 2015;37:48-54.
[14] Wu Z, Rademakers T, Kiessling F, Vogt M, Westein E, Weber C, et al. Multiphoton microscopy in cardiovascular research. Methods 2017;130:79-89.

[15] Sacks MS. Small angle light scattering methods for soft connective tissue structural analysis. In: Encyclopedia of biomaterials and biomedical engineering. CRC Press; 2008. p. 2450-63.

[16] Gaul R, Nolan D, Lally C. Collagen fibre characterisation in arterial tissue under load using SALS. J Mech Behav Biomed Mater 2017;75:359-68.

[17] Gasparotti E, Vignali E, Losi P, Scatto M, Fanni BM, Soldani G, et al. A 3d printed melt-compounded antibiotic loaded thermoplastic polyurethane heart valve ring design: an integrated framework of experimental material tests and numerical simulations. Int J Polym Mater Polym Biom 2019;68(1-3):1-10.

[18] Auricchio F, Ferrara A, Lanzarone E, Morganti S, Totaro P. A regression method based on noninvasive clinical data to predict the mechanical behavior of ascending aorta aneurysmal tissue. IEEE Trans Biomed Eng 2016:64(11):2607-17.

[19] Celi S, Losi P, Berti S. Investigation on regional variation of intraluminal thrombus: a mechanical and histological study. Bioinspired Biom Nanobiom 2012;1(3):183-94.

[20] Celi S, Berti S. Biomechanics and fe modelling of aneurysm: review and advances in computational models. INTECH Open Access Publisher; 2012.

[21] O'Leary SA, Doyle BJ, McGloughlin TM. The impact of long term freezing on the mechanical properties of porcine aortic tissue. J Mech Behav Biomed Mater 2014;37:165-73.

[22] Vande Geest JP, Sacks MS, Vorp DA. Age-related differences in the biaxial biomechanical behavior of human abdominal aorta. In: Proceedings of the ASME international mechanical engineering congress and exposition, 36509; 2002. p. $115-16$

[23] Di Puccio F, Celi S, Forte P. Review of experimental investigations on compressibility of arteries and introduction of a new apparatus. Exp Mech 2012;52(7):895-902.

[24] Linder T, Löfquist T, Wernersson EL, Gren P. Light scattering in fibrous media with different degrees of in-plane fiber alignment. Opt Express 2014;22(14):16829-40.

[25] Robitaille MC, Zareian R, DiMarzio CA, Wan K-T, Ruberti JW. Small-angle light scattering to detect strain-directed collagen degradation in native tissue. Interface Focus 2011:1(5):767-76.

[26] Niestrawska JA, Ch Haspinger D, Holzapfel GA. The influence of fiber dispersion on the mechanical response of aortic tissues in health and disease: a computational study. Comput Methods Biomech Biomed Engin 2018;21(2):99-112.

[27] Babu AR, Byju AG, Gundiah N. Biomechanical properties of human ascending thoracic aortic dissections. J Biomech Eng 2015;137(8).

[28] Gaul R, Nolan D, Ristori T, Bouten C, Loerakker S, Lally C. Strain mediated enzymatic degradation of arterial tissue: insights into the role of the non-collagenous tissue matrix and collagen crimp. Acta Biomater 2018;77:301-10.

[29] Gasser TC, Gallinetti S, Xing X, Forsell C, Swedenborg J, Roy J. Spatial orientation of collagen fibers in the abdominal aortic Aneurysm's wall and its relation to wall mechanics. Acta Biomater 2012;8(8):3091-103.

[30] Watson SR, Liu P, Peña EA, Sutton MA, Eberth JF, Lessner SM. Comparison of Aortic Collagen Fiber Angle Distribution in Mouse Models of Atherosclerosis Using Second-Harmonic Generation (SHG) Microscopy. Microsc Microanal 2016;22(1):55-62.

[31] Vignali E, Martini N, Losi P, Capellini K, Della Latta D, Di Bartolo F, et al. Towards non-invasive assessment of human aortic tissue microstructure: diffusion tensor imaging for collagen fiber detection. Sixth National Congress of Bioengineering; 2018. p. 156-9.

[32] Tornifoglio B, Stone AJ, Johnston RD, Shahid SS, Kerskens C, Lally C. Diffusion tensor imaging and arterial tissue: establishing the influence of arterial tissue microstructure on fractional anisotropy, mean diffusivity and tractography. bioRxiv; 2020. doi:101101/20200520104711.

[33] de Beaufort HW, Ferrara A, Conti M, Moll FL, van Herwaarden JA, Figueroa CA, et al. Comparative analysis of porcine and human thoracic aortic stiffness. Eur J Vas Endovas Surgery 2018;55(4):560-6.

[34] Cavinato C, Helfenstein-Didier C, Olivier T, Du Roscoat SR, Laroche N, Badel P. Biaxial loading of arterial tissues with $3 \mathrm{~d}$ in situ observations of adventitia fibrous microstructure: a method coupling multi-photon confocal microscopy and bulge inflation test. J Mech Behav Biomed Mater 2017;74:488-98.

[35] Capellini K, Vignali E, Costa E, Gasparotti E, Biancolini ME, Landini L, et al. Computational fluid dynamic study for aTAA hemodynamics: an integrated image-based and radial basis functions mesh morphing approach. J Biomech Eng 2018;140(11).

[36] Capellini K, Gasparotti E, Cella U, Costa E, Fanni BM, Groth C, et al. A novel formulation for the study of the ascending aortic fluid dynamics with in vivo data. Med Eng Phys 2020. doi:10.1016/j.medengphy.2020.09.005. 